\title{
Promoção da saúde e estilo de vida em
}

\section{tempos de GOVID-19}

\author{
Ana Luísa Patrão \\ Sheila Alvim ${ }^{2}$ \\ Francisco Pitanga ${ }^{3}$ \\ Ciro Oliveira Queiroz ${ }^{4}$ \\ Rogério Tosta de Almeida ${ }^{5}$ \\ Yadira Morejón Terán ${ }^{6}$
}

1 Doutora em Psicologia da Saúde (Portugal) e pós-doutora em Saúde Coletiva (Brasil).Professora Colaboradora do Instituto de Saúde Coletiva (ISC) da Universidade Federal da Bahia (UFBA) e Pesquisadora da Faculdade de Psicologia e de Ciências da Educação da Universidade do Porto (Portugal). Atua na linha de pesquisa sobre o estudo dos fatores psicossociais e culturais, bem como questões de gênero, na promoção da saúde e saúde pública.

2 Nutricionista. Doutora em Saúde Pública, professora associada e docente do Programa de PósGraduação em Saúde Coletiva (PPGSC) da Universidade Federal da Bahia (UFBA). Coordenadora do ELSA-Brasil da UFBA. Atuação nas temáticas da saúde do adulto e de crianças principalmente doenças crônicas não transmissíveis e padrões alimentares.

3 Profissional de Educação Física. Doutor em Saúde Coletiva, professor associado da Universidade Federal da Bahia (UFBA). Atuação nas temáticas das doenças crônicas não transmissíveis, atividade física e sedentarismo.

4 Profissional de Educação Física. Doutor em Medicina e Saúde Humana. Professor Adjunto da Escola Bahiana de Medicina e Saúde Pública. Atuação nas temáticas da epidemiologia da atividade física, inter-relação atividade física, aptidão física e saúde..

5 Profissional de Educação Física. Doutor em Saúde Pública. Professor adjunto da Universidade Estadual de Feira de Santana (UEFS). Atuação nas temáticas da epidemiologia da atividade física e de Doenças Crônicas Não Transmissíveis (DCNT).

6 Nutricionista (Equador). Doutora em Saúde Pública, vice-coordenadora do Estudio de Factores de Riesgo Cardiometabolico en Adultos Ecuatorianos (Efrica). Atuação nas temáticas do consumo alimentar e Doenças Crônicas Não Transmissíveis (DCNT).

PATRÃO, A. L.; ALVIM, S.; PITANGA, F.; QUEIROZ, C. O.; ALMEIDA, R. T. de; MOREJÓN-TERÁN, Y. Promoção da saúde e estilo de vida em tempos de COVID-19. In: BARRETO, M. L.; PINTO JUNIOR, E. P.; ARAGÃO, E.; BARRAL-NETTO, M. (org.). Construção de conhecimento no curso da pandemia de COVID-19: aspectos biomédicos, clínico-assistenciais, epidemiológicos e sociais. Salvador: Edufba, 2020. v. 2. DOI: https://doi.org/10.9771/9786556300757.012 


\section{Introdução}

Em tempos de pandemia, é essencial que façamos todo o possível para nos mantermos saudáveis. A prioridade tem sido seguir as recomendações das entidades de saúde relativas à prevenção da transmissão da COVID-19, tais como distanciamento social, etiqueta respiratória e higienização das mãos e espaços. (WHO, 2020b) No entanto, é igualmente importante não negligenciar os comportamentos de saúde que definem um estilo de vida saudável, essenciais para a promoção da saúde física e psicológica da população em geral, mas especialmente para quem já possui fatores de risco relevantes para as doenças crônicas. (VAN DEN BROUCKE, 2020)

A promoção da saúde baseia-se na aceitação de que os comportamentos em que nos envolvemos têm impacto e são responsáveis pela nossa saúde (BENNET; MURPHY, 1999), chamados assim de comportamentos de saúde relacionados ao estilo de vida. Quatro comportamentos em particular são especialmente associados a um conjunto de doenças crônicas atuais:

1. a ausência de atividade física/aumento do comportamento sedentário;

2. a alimentação inadequada; 
3. o abuso de álcool; e

4. o consumo de tabaco. (OGDEN, 2004; PATRÃO et al., 2019b)

A evidência da relação entre comportamento - sobretudo estes quatro em particular - e a saúde é, atualmente, inegável (BENNET, 2002), o que leva a outra constatação importante: se a saúde depende em grande parte dos comportamentos, então, se adequadamente estimulados por estratégias de saúde pública (PEARCE et al., 2014), é passível de ser melhorada também pela ação do próprio indivíduo e dos seus grupos, através da modificação dos fatores que a influenciam. (PATRÃO et al., 2017) A verdade é que, qualquer lugar do mundo, desde o mais democrático ao mais autocrático e autoritário, ou, do mais desenvolvido até ao mais em vias de desenvolvimento, teve que lidar com a realidade que se impôs: a pandemia da COVID-19, que obrigou à responsabilidade social do confinamento e do distanciamento físico. (WHO, 2020b) Dessa forma, os domicílios se constituíram no local de maior tempo de permanência. Populações em todo o mundo experimentaram mudanças temporárias nos modos de viver relacionadas ao trabalho, lazer e família, com consequências para o estilo de vida. Diante desse contexto, as pessoas assumiram comportamentos que podem ser divergentes daqueles até então praticados, especialmente com relação aos quatro supracitados. Por exemplo, deu-se uma maior exposição às propagandas/programas de TV (filmes e séries) e redes sociais e, consequente, mais consumo de alimentos ultraprocessados e preparação de refeições mais calóricas - preparações em casa de bolos, pães, pizzas com farinhas refinadas -; mais comportamento sedentário pelo uso de telas associado a uma menor mobilidade física devido ao confinamento; e mais consumo de álcool e tabaco, quer como coping para lidar com o estresse, quer pela sensação de disponibilidade de tempo e espaço para comportamentos que até então 
eram circunscritos a momentos próprios da semana - final de semana, apenas numa festa etc.

Posto isso, neste capítulo, através de uma revisão da literatura científica, pretendemos discorrer sobre os aspectos da promoção da saúde durante a pandemia e os períodos de confinamento, bem como analisar as diferenças e particularidades de gênero a esse nível.

\section{Atividade física e comportamento sedentário}

A definição de atividade física como qualquer movimento corporal produzido pela musculatura esquelética que resulte em gasto energético acima do metabolismo de repouso (CASPERSEN; POWELL; CHRISTENSON, 1985), ainda muito utilizada, foi estrategicamente importante para as pesquisas relacionadas à saúde e para se pensar em ferramentas de mensuração. Entretanto, a atividade física enquanto comportamento apresenta-se como um fenômeno complexo e as suas definições e formas de mensuração são diversas. Um conceito mais recente, que considera a atividade física para além dos aspectos fisiológicos, aponta que existem outras qualidades inerentes a sua prática que precisam ser reconhecidas e consideradas, como, por exemplo, os processos psicológicos, as condições sociais, culturais e políticas. Assim, segundo Piggin (2020, p. 5, tradução nossa), “a atividade física envolve pessoas se movendo, agindo e atuando em espaços e contextos culturalmente específicos e influenciados por uma gama única de interesses, emoções, ideias, instruções e relacionamento".

Os estudos sobre os efeitos do comportamento sedentário na saúde das pessoas são mais recentes. Tremblay e demais autores (2017) localizaram na literatura, pelo menos, 12 definições diferentes para o termo, sinalizando a necessidade de uma definição operacional padronizada. Assim, um conceito mais recente e relativamente 
consensual, considera o comportamento sedentário como uma atividade com gasto energético abaixo de 1,5 equivalentes metabólicos, incluindo os comportamentos de sentar, reclinar ou deitar, excetuando-se as horas de sono, para realizar atividades de ler, estudar, assistir televisão, usar o computador, entre outras. (PATE; O'NEILL; LOBELO, 2008; TREMBLAY et al., 2017) Além disso, ao se fazer a análise da origem da palavra "sedentário", observa-se que a mesma vem do latim sedere, que significa sentado. Assim, percebe-se que classificar como sedentário quem não pratica atividade física não é o mais indicado. A terminologia adotada pelo International Physical Activity Questionnaire (IPAQ) para a classificação do nível de atividade física de pessoas que não realizam nenhuma atividade física por pelo menos 10 minutos contínuos durante a semana como "sedentários", pode, dentre outros fatores, ter contribuído para esse equívoco. Atualmente, sabe-se que se a pessoa cumpre a recomendação da Organização Mundial da Saúde (OMS) de fazer pelo menos 150 minutos de atividade física por semana, mas, além disso, fica muitas horas sentado, reclinado ou deitado durante o dia, pode-se dizer que essa pessoa apesar de ser ativa fisicamente também temo comportamento sedentário. Diferentes autores vêm demonstrando que a junção desses dois comportamentos, ou seja, ser ativo fisicamente e reduzir os comportamentos sedentários pode trazer importantes benefícios para a saúde cardiometabólica, bem como para redução da mortalidade por todas as causas. (CHAU et al., 2014; MORAES et al., 2013; HAMER; STAMATAKIS; STEPTOE, 2014; PITANGA et al., 2019; VAN DER PLOEG et al., 2012)

No contexto da pandemia da COVID-19, alguns autores e diversas instituições governamentais, sociedades, associações médicas, conselhos profissionais e instituições científicas publicaram documentos sugerindo a manutenção da prática da atividade física e redução do comportamento sedentário, justificando essa recomendação pelo reconhecimento da comunidade científica dos benefícios 
proporcionados para a saúde cardiovascular, metabólica e mental, bem como para o sistema imunológico. (AMERICAN COLLEGE OF SPORTS MEDICINE, 2020; CHEN et al., 2020; JONSDOTTIR et al., 2010; KNUTH; CARVALHO; FREITAS, 2020; KRINSKI et al., 2010; LIN et al., 2016; PITANGA; BECK; PITANGA, 2020a) Alguns autores sugeriram ainda que a atividade física fosse considerada como essencial durante a pandemia. (PITANGA; BECK; PITANGA, 2020c) Entretanto, há controvérsias sobre o assunto. (ALMEIDA; QUEIROZ; AQUINO, 2020; CARVALHO; SILVA; OLIVEIRA, 2020) Nesse sentido, desde o início da pandemia foram sugeridas a realização de atividades físicas no ambiente domiciliar, preferencialmente, orientadas por profissionais de educação física de forma remota, bem como quando autorizada pelos órgãos governamentais, a prática de atividades físicas ao ar livre, como caminhadas, corridas e andar de bicicleta, feitas de forma individual e com uso de máscaras faciais, evitando-se aglomerações e seguindo-se todos os protocolos de biossegurança recomendados pelas autoridades sanitárias. (AMERICANCOLLEGE OF SPORTS MEDICINE, 2020; CHEN et al., 2020; JIMÉNEZ-PAVÓN; CARBONELLBAEZA; LAVIE, 2020; PITANGA; BECK; PITANGA, 2020a) De fato, estudos que avaliam a eficácia e segurança de exercícios minimamente monitorados não são novos. Em metanálise realizada com dados de estudos de treinamento contra resistência com indivíduos idosos, foi demonstrado que, embora o desempenho e função muscular sejam maiores quando a supervisão dos exercícios se dá presencialmente, o programa minimamente supervisionado em casa, com baixo número de visitas, foi efetivo e seguro. (KIS et al., 2019) Provavelmente, futuros estudos, realizados durante e pós-pandemia de COVID-19, apontem na mesma direção para a supervisão a distância.

Percebe-se ainda que a atividade física foi muito evidenciada nos discursos das entidades médicas, públicas e corporativas e com predomínio para as respostas biológicas. Entretanto, algumas 
condutas governamentais de acionar indivíduos e famílias a realizarem atividade física sem uma maior instrumentalização, apoio e acesso a profissionais, fortalecem ainda mais a lógica do mercado que centra no indivíduo a responsabilidade sobre sua saúde e não considera as condições ambientais, econômicas e culturais da população. (CARVALHO; SILVA; OLIVEIRA, 2020; KNUTH; CARVALHO; FREITAS, 2020)

Existem várias alternativas de práticas de atividades físicas para serem realizadas no ambiente familiar e domiciliar, principalmente em indivíduos portadores de cardiopatias (PITANGA; BECK; PITANGA, 2020), porém, as pessoas precisam ser estimuladas e orientadas. Muitos profissionais de educação física já utilizam mensagens de telefones celulares, aplicativos, e-mails ou vídeochamadas para prescrever e monitorar o exercício individualmente (OLIVEIRA NETO et al., 2020), assim como existem livres iniciativas de orientações de atividades físicas na internet, muitas delas ligadas às universidades ou a entidades não governamentais, como o guia da OMS (WHO, 2020c), por exemplo, que poderiam ser implementadas por governos, com campanhas educativas de massa, ampliando o alcance dessa mensagem gratuitamente para toda a população. Nessa direção, Solon Júnior e demais autores (2020) demonstraram que aqueles indivíduos que mantiveram sua atividade física em casa durante a pandemia apresentaram melhor qualidade de vida, qualidade subjetiva de sono e níveis reduzidos de ansiedade, estresse e depressão, em comparação com aqueles indivíduos que não se exercitaram.

Apesar de todas essas recomendações, notou-se que a prática da atividade física foi reduzida durante o período da pandemia, fato que pode impactar negativamente nos níveis de aptidão física. (PINHO et al., 2020) Dados do FITBIT (2020) relataram diminuição no número de passos diários na população mundial, sendo que no Brasil chegou a 15. Corroborando com esses achados, 
Malta e demais autores (2020) demonstraram que 31,1\% (IC95\%: 28,9-31,5) dos adultos brasileiros que realizavam atividade física suficiente antes da COVID-19, durante a pandemia, esse percentual reduziu para apenas 12,0\% (IC95\%: 11,1-12,9). Além disso, o comportamento sedentário - tempo assistindo TV - aumentou em 1 hora e 45 minutos em relação ao tempo médio antes da pandemia. Assim, estamos diante de um cenário em que a pandemia da COVID-19 pode causar redução da atividade física, configurando um quadro de múltiplas pandemias, já que a inatividade física também é considerada uma pandemia, responsável por mais de 3 milhões de mortes por ano ao redor mundo. Todas essas evidências nos remetem para um quadro alarmante, com várias pandemias simultâneas: COVID-19, inatividade física e obesidade, convivendo e se retroalimentando mutuamente. (NIEMAN, 2020; PITANGA; BECK; PITANGA, 2020b)

Apesar de não haver ainda estudos que demonstrem a importância da prática de atividade para a prevenção de mortalidade, ou mesmo para a prevenção de quadros clínicos mais graves da COVID19, diversas pesquisas foram publicadas demonstrando esses efeitos em relação à influenza/H1N1, além de evidenciarem também o efeito da atividade física para melhor efetividade da vacina contra a influenza/H1N1. (KOHUT et al., 2002; SCHULER; LEBLANC; MARZILLI, 2003; SIU; CAMPITELLI; KWONG, 2012; WONG et al., 2008; WOODS et al., 2009) Nesse sentido, alguns autores sugerem que esses resultados podem ser transportados para a COVID-19, já que se trata de infeções virais com características semelhantes. (LUZI; RADAELLI, 2020) Assim, a comunidade científica mundial vem alertando para a importância da adoção de hábitos saudáveis, entre eles, a manutenção da prática de atividade física durante e após a pandemia para que assim as pessoas possam estar mais bem preparadas para a atual, bem como, para futuras outras pandemias com características similares. (LADDU et al., 2020) 
Dessa forma, seria muito importante o estímulo da prática de atividade física, para todas as idades e gêneros, em ambientes domiciliares, ao ar livre ou em diferentes ambientes apropriados para tal (CORTEZ et al., 2020), respeitando as orientações das autoridades sanitárias e determinações governamentais para manutenção do distanciamento social, e, sempre que possível, com orientação de um profissional de educação física. Isso seria uma mensagem de saúde pública importante para a manutenção da saúde física e mental e enfrentamento desse momento de ansiedade generalizada e sensação de desamparo e, que, portanto, deve ser mais bem difundida e veiculada.

\section{Comportamento alimentar}

O "comportamento alimentar" é um termo que inclui a motivação para adoção de determinadas práticas alimentares e os problemas associados a estas. Baseia-se nesse conceito as propostas de promoção da alimentação saudável, prevenção de problemas de saúde e intervenções. (LACAILLE et al., 2013) Esses comportamentos são também influenciados pelo grau de escolaridade/ renda e, quando repetidos ou validados pelo grupo social ao qual os indivíduos pertencem, podem se tornar hábitos diários reforçados nas experiências anteriores pela exposição a diversos ambientes alimentares, desde a busca por alimentos até a forma de ingestão. (MARIJN STOK et al., 2018) Com o surgimento da COVID-19, todas essas questões motivacionais, comportamentais e circunstanciais da alimentação foram desafiadas e alteradas.

Embora ficar em casa e praticar o distanciamento social sejam necessários para prevenção da transmissão da COVID-19 e evitar o colapso dos sistemas sanitários, ele gerou impactos de diversas ordens em aspectos que interferiram no comportamento 
alimentar das populações. (DI RENZO et al., 2020a) A restrição das atividades do comércio, restaurantes, interdição ou restrição de praças/serviços de alimentação, entre outras, alterou os padrões de consumo de bens e serviços, rotinas de compra e preparação dos alimentos, e mudou os hábitos alimentares nas populações ao proporcionar o consumo de alimentos não saudáveis. (AMMAR et al., 2020) Contudo, o ato de cozinhar tornou-se mais frequente durante o confinamento e o maior nível educacional materno esteve associado com o maior consumo de frutas e vegetais por adolescentes participantes de um estudo conduzido na Itália, Espanha, Chile, Colômbia e Brasil. (RUIZ-ROSO et al., 2020)

No âmbito das mudanças dos comportamentos, nos Estados Unidos, há registro de aumento da ordem de $25 \%$ no consumo de aplicativos de instalação de TV. (NIELSEN, 2020) Brasileiros também tiveram seu tempo de assistência à TV aumentado em 1h e 45min após o início da epidemia. (MALTA et al., 2020) Isso possivelmente se somou às dificuldades inerentes ao distanciamento social para a promoção de comportamentos alimentares saudáveis, uma vez que o conteúdo da TV e redes sociais pode estimular a adoção de comportamentos alimentares não saudáveis. (BORZEKOWSKI; ROBINSON, 2001; LEE et al., 2014) O maior tempo de exposição às redes e propagandas da indústria de alimentos é um estímulo ao consumo de alimentos ultraprocessados, especialmente aquelas dirigidas às crianças e adolescentes. (CLARKE, 2020; THE NIELSEN COMPANY, 2020) Esse grupo etário é particularmente susceptível às propagandas pela incapacidade de julgamento e inexperiência (HARTUNG; KARAGEORGIADIS, 2017), e à força do grupo social para que sejam aceitos. Por outro lado, também está sujeito à ausência do ambiente escolar, livre de publicidade garantido por lei, na alimentação das crianças como promotora de hábitos alimentares saudáveis (RUNDLE et al., 2020) e à dificuldade dos pais/cuidadores em acompanhar as atividades 
das crianças no trabalho em casa (home office). (DEL BOCA et al., 2020)

Contudo, o efeito do distanciamento social nos comportamentos alimentares apenas deu maior visibilidade aos problemas alimentares prévios à pandemia. A coexistência da obesidade com a fome e carência de micronutrientes vem-se a converter na maior ameaça nutricional nos últimos anos (DURAN; CABALLERO; ONIS, 2006) e, nesse cenário, o excesso de peso é observado na presença de dietas ultraprocessadas, hipercalóricas, com alto teor de gordura saturadas, carboidratos refinados e açúcares, baixo conteúdo de fibra e gorduras insaturadas (CORDAIN et al., 2005), mas deficientes em minerais e vitaminas. (LÓPEZ DE ROMAÑA; OLIVARES; BRITO, 2015) Essa seleção alimentar está associada com a obesidade, um fator de risco importante para a COVID-19 (DIETZ; SANTOSBURGOA, 2020), havendo o encontro dessas duas pandemias neste momento. (KHAN; MOVERLEY SMITH, 2020) Esses achados trazem com maior força a importância do controle da obesidade e problemas associados antes e após a pandemia, por meio da promoção de comportamentos alimentares saudáveis e prudentes.

É possível que o confinamento tenha levado a mudanças nos comportamentos alimentares também por medo e ansiedade. Macth (2008) observou que esses comportamentos são afetados pelas condições de estresse, angústia e transtornos emocionais, adquirindo padrões alimentares pouco saudáveis e de baixa qualidade. No início da pandemia, registraram-se informações que levaram ao pânico e compra excessiva de alimentos e produtos, interrompendo o abastecimento dos mercados. (NAJA; HAMADEH, 2020; SPENCER, 2020) O confinamento e a dependência das indústrias dos produtos alimentícios importados durante a pandemia expuseram ainda mais a fragilidade da agricultura e economia dos países. A importância da agroecologia vem sendo discutida para melhorar a saúde ambiental e humana (ALTIERI; NICHOLLS, 2020), 
na procura de uma maior diversidade nos cultivos e na dieta das populações.

A crise sanitária trouxe dificuldades para os pequenos agricultores com o fechamento das feiras livres e com inclinação da população para compra de produtos ultraprocessados para estoque. Contudo, no Brasil, foi possível observar a mobilização de pequenos produtores de alimentos orgânicos e agroecológicos na organização de entregas à domicílio ou entregas de "cestas" compradas pela internet em pontos específicos das cidades, garantindo o fornecimento de alimentos frescos e saudáveis, infelizmente apenas a uma parcela pequena da população. (PEIXOTO; LANDAU, 2020; RAMOS, 2020) As iniquidades sociais limitam a possibilidade de adoção e manutenção de comportamentos alimentares saudáveis, o que se tornou ainda mais difícil no contexto da pandemia. Nas primeiras três semanas de quarentena no Reino Unido, foi 4,1 vezes mais provável que pessoas de menor posição socioeconômica não conseguissem acesso a alimentos de forma suficiente na semana 1 , e 4,9 vezes na semana 3 , comparadas àquelas de mais alta posição socioeconômica. (WRIGHT; STEPTOE; FANCOURT, 2020)

No âmbito das estratégias para promoção da alimentação saudável durante a pandemia, a OMS divulgou para o público europeu Food and nutrition tips during self-quarantine com dicas sobre os alimentos mais recomendados para consumo durante o distanciamento social, com destaque para o consumo de legumes, vegetais e frutas. (WHO, 2020a) No Brasil, o guia alimentar para a população brasileira (BRASIL, 2014) é um avançado instrumento para a promoção da alimentação saudável e traz dicas de combinações saudáveis de alimentos de forma a evitar alimentos ultraprocessados (MONTEIRO et al., 2019), e que serve como guia para melhor alimentação antes, durante e após a pandemia. 
O engajamento dos profissionais da saúde e de toda a sociedade na consideração das recomendações da Organização das Nações Unidas para a Alimentação e a Agricultura (FAO) e OMS e das diretrizes nacionais para alimentação saudável, redução e controle da publicidade de alimentos e bebidas ultraprocessados e, sobretudo, a redução das desigualdades sociais e de gênero, são aspectos fundamentais para que exista o estímulo à escolha certa de um comportamento alimentar que envolva alimentos com menor nível de processamento, preparados e consumidos por toda a família. (MONTEIRO et al., 2019; RUNDLE et al., 2020) Essas recomendações devem ser accessíveis e inclusivas à sociedade, sendo que políticas públicas de promoção da saúde apresentam benefícios à longo prazo na prevenção de doenças, incluindo a COVID19, e a redução da obesidade, o que inclusive poderia aumentar a efetividade das vacinas em pessoas com essa condição. (GREEN; BECK, 2017)

\section{Consumo de álcool e tabaco}

O comportamento de beber e fumar como estratégia de enfrentamento (coping) para lidar com situações estressantes está bastante reportado na literatura (GARCÍA-ÁLVAREZ et al., 2020; LEBEAUT; TRAN; VUJANOVIC, 2020; PATRÃO et al., 2019a), principalmente em situações em que há falta de suporte social e emocional. (TAKEDA et al., 2004; TORRES; NOWSON, 2007) Com o surgimento da pandemia por COVID-19 e a necessidade de a população mundial adotar estratégias de isolamento social, observou-se um aumento dos problemas de saúde mental associados ao consumo de substâncias, entre as quais as lícitas como são o álcool e o tabaco. (VERMA; MISHRA, 2020) 
Na China e Europa, os locais onde primeiramente se reportou a pandemia, há alguns estudos que relatam um aumento desses consumos. Na China, Sun e demais autores (2020) verificaram que, desde o início da pandemia, o aumento do consumo de álcool e tabaco foi relatado por 32\% dos bebedores regulares e $20 \%$ dos fumantes regulares, $19 \%$ dos ex-bebedores e $25 \%$ dos ex-fumantes relataram recaída, e apenas 3\% dos bebedores regulares e $8 \%$ dos fumantes regulares pararam de beber e fumar. Em contexto europeu, um estudo populacional croata revelou que, durante os dez dias do confinamento em que ocorreu o inquérito on-line, o número de cigarros fumados aumentou significativamente (de 11,8 \pm 7,4 cigarros/dia antes do bloqueio para 13,9 $\pm 9,8$ cigarros/dia durante o bloqueio), bem como o consumo de álcool, sobretudo o que se referia a beber mais de sete bebidas alcoólicas por semana. (ĐOGAŠ et al., 2020) No mesmo sentido, um estudo desenvolvido por Jackson e demais autores (2020), na Inglaterra, revelou que o confinamento no mês de março foi associado a aumentos na prevalência de consumo de álcool de alto risco (38,3\% antes de março versus $25,1 \%$ depois de março). Em Itália, um estudo de Caponnetto e demais autores (2020) revelou que, embora tenha havido uma intenção de largar de fumar por parte da maioria dos fumantes convencionais, as pessoas que fumam cigarro eletrónico não revelaram qualquer intenção de o fazer e ex-fumantes mostraram intenção de voltar a fumar na fase de confinamento. Também na Itália, Cancello e demais autores (2020) verificaram que comparando o pré com o pós-confinamento no norte do país, 38\% dos fumantes aumentaram significativamente o consumo de cigarros. Na Polônia, um estudo de Sidor e Rzymski (2020) revelou um aumento no consumo de álcool em $14,6 \%$ da amostra, com maior tendência para pessoas com problemas com este tipo de consumo, e também um aumento na frequência de fumar durante a quarentena. 
No Brasil, um estudo de base populacional, desenvolvido por Malta e demais autores (2020), mostrou que, no que se refere ao consumo de tabaco, 34\% dos fumantes relataram ter aumentado o consumo de cigarros durante a pandemia: aumento de 10 cigarros por dia foi observado em 22,5\% (IC95\%: 19,6;25,7), e de 20 cigarros por dia, em 5,1\% (IC95\%: 3,4;7,7) dos participantes. No que se refere ao maior consumo de álcool durante o confinamento, este foi reportado por $17,6 \%$ (IC95\%: 16,4;18,9), sem diferenças por sexo. A maior prevalência do consumo de álcool foi encontrada sobretudo entre adultos e jovens, nas pessoas de 30 a 39 anos de idade (24,6\% - IC95\%: 21,2;28,3), seguidas daquelas entre 18 e 29 anos. O grupo específico dos médicos e profissionais de saúde, submetidos a níveis especiais de exposição e, portanto, sobre uma grande pressão, também revelou consumos mais elevados de álcool e tabaco. Também no Brasil, um estudo com 766 urologistas revelou que, acompanhado de uma redução drástica do número de consultas e cirurgias agendadas, surgiu um aumento significativo do consumo de álcool, com 39,9\% desta amostra referindo que passou a consumir mais álcool durante o mês de abril de 2020. (GOMES et al., 2020)

No que se refere especificamente ao ato de fumar, surgem alguns estudos cujos resultados seguem no sentido oposto dos apresentados anteriormente. Por exemplo, o estudo de Jackson e demais autores (2020), com uma amostra populacional de aproximadamente 20 mil adultos, revelou que o evento de COVID-19 não foi associado a uma mudança significativa na prevalência de tabagismo (17,0\% depois versus $15,9 \%$ antes), mas foi associado a aumentos na taxa de abandono e de tentativas (39,6\% versus $29,1 \%$,), bem como de cessação efetiva ( $8,8 \%$ versus $4,1 \%)$. O estudo de Di Renzo e demais autores (2020a) com mais de 3 mil italianos também revelou que 3,3\% dos fumantes inquiridos haviam cessado o hábito de fumar durante o confinamento. Outro estudo 
italiano de Caponnetto e demais autores (2020) mostrou que a maioria dos fumantes de cigarro convencional (não eletrônico) pensou em cessar o consumo. Ou seja, dependendo do estudo e do contexto, os resultados a este nível variam muito. Por exemplo, o estudo de Yach (2020), no âmbito do seu trabalho desenvolvido na Foundation for a Smoke-Free World, reporta os resultados de uma pesquisa ocorrida em vários países - Itália, Índia, África do Sul, Reino Unido e Estados Unidos - em simultâneo durante o mês de abril de 2020, época de confinamento, revelando que o consumo de cigarro eletrônico aumentou marginalmente em todos. No entanto, quando analisados os resultados por país, percebeu-se que na Índia, uma grande parte da população pesquisada acredita que fumar e vaporizar aumenta o risco de contrair COVID-19. Já na Itália, mais da metade acredita que não há relação entre o risco de COVID-19 e tabagismo ou cigarro eletrônico. Os resultados de outros países foram menos evidentes, embora vaporizar era geralmente considerado um risco menor do que fumar cigarros convencionais. Em uma grande proporção de usuários, o desejo de parar de fumar ou vaporizar por razões de saúde foi afetado pela experiência direta do surto de COVID-19. No Reino Unido e nos Estados Unidos, essa proporção foi duas vezes maior entre os participantes que viviam em uma casa onde alguém havia testado positivo para o vírus.

No que se refere aos fatores associados ao aumento desses consumos resultante de estresse relacionado com eventos de saúde, um dos raros estudos que existe sobre o tema foi desenvolvido na China quando da epidemia de SARS. Nesse caso, o aumento da dependência de álcool foi associado a ter trabalhado como uma equipe de saúde durante a epidemia, mesmo três anos após o surto, especificamente, o grau de exposição ao vírus e ter ficado isolado. Verificou-se uma relação dose-resposta entre a intensidade 
da exposição ao vírus e o abuso e dependência de álcool de longo prazo. (WU et al., 2008)

Há a realçar que, embora a questão do estresse, depressão, ansiedade seja preponderante na hora de explicar o aumento do consumo de álcool e tabaco, poderão haver outras explicações para esses comportamentos, nomeadamente, o tédio associado à permanência em casa (GARCÍA-ÁLVAREZ et al., 2020) ou ainda o facto da casa ter passado a ser local onde tudo ocorre na vida das pessoas, incluindo o lazer. Especificamente, as pessoas passaram a beber e fumar em casa, comportamentos que até então poderiam ser apenas sociais para muitos. No entanto, fazê-lo fora do contexto e em circunstâncias anormais em termos de horários poderá ter contribuído para o seu aumento. O confinamento parcial ou total imposto pela luta contra a COVID-19, que poderá repetir-se em alguns locais e até novamente em todo o mundo, deve ser acompanhado de medidas que tenham em atenção o aumento dos consumos de álcool e tabaco. Por serem consumos lícitos na grande maioria das sociedades mundiais e em todas as ocidentais, os consumos exacerbados de álcool e o consumo de tabaco, responsáveis pelo agravamento e desenvolvimento de algumas das doenças crônicas mais letais (OGDEN, 2004; PATRÃO et al., 2019b), passam, frequentemente despercebidos e, em momentos de crise pandêmica, como a que estamos vivendo, isso ocorre ainda de forma mais acentuada. Tal não poderá acontecer pois, concordando com o que o The Lancet Gastroenterology Hepatology (2020) tem vindo a alertar, os problemas de saúde globalmente significativos que existiam antes da COVID-19 - e para os quais o consumo de álcool e tabaco concorrem grandemente - não desapareceram, pelo contrário, em muitos casos a pandemia poderá tê-los exacerbado. Assim, considera-se de extrema importância que os programas de promoção da saúde relacionados com o consumo de álcool e tabaco só não sejam descurados, como sejam 
intensificados, no sentido de ajudar as pessoas a não iniciarem, nem exacerbarem esses consumos.

\section{Diferenças de gênero ao nível dos comportamentos de saúde e estilo de vida}

A literatura fornece evidências abundantes sobre a influência preponderante do gênero na saúde em geral, e nos comportamentos de saúde em particular. (HANKIVSKY, 2012; PATRÃO et al., 2017; READ; GORMAN, 2010) No que se refere especificamente aos comportamentos preventivos face à COVID-19, as mulheres tendem a cumprir mais seriamentente as recomendações de saúde. (GALASSO et al., 2020) Já no que se refere especificamente aos comportamentos de saúde relacionados ao estilo de vida, estes perpassam uma série de circunstâncias pessoais e sociais mais abrangentes, que se acentuam em tempos de COVID-19.

A prevalência da prática de atividade física no Brasil entre os homens (33\% - IC95\%; 30,7-35,5) já era maior antes da pandemia em comparação com as mulheres (27,6\% - IC95\%; 26,229,0 ). Durante a pandemia, ambos os sexos reduziram sua prática, entretanto, os homens se mantiveram em maior proporção $(14,0 \%$ - IC95\%; 12,4-15,8), em comparação às mulheres (10,3\% - IC95\%; 9,4-11,2). Com relação ao comportamento sedentários, homens e mulheres aumentaram durante a pandemia, em proporção similar. (MALTA et al., 2020) Em estudo realizado em cidade do sul do Brasil, verificou-se uma alta desigualdade na prática de atividade física entre os sexos, com os homens apresentando 20 pontos percentuais maior do que as mulheres. (CROCHEMORESILVA et al., 2020) Em outra investigação realizada na cidade do Rio de Janeiro também foi observado que os indivíduos do sexo 
masculino tinham uma maior chance de praticar atividade física durante o distanciamento social. (RODRIGUES et al., 2020) Esses dados nos mostram que parece haver diversas barreiras para as mulheres praticarem atividade física de lazer, desde os afazeres domésticos, cuidado de pessoas em casa e atividades do próprio trabalho. Ou seja, as velhas desigualdades de gênero que afetam o tempo de lazer das mulheres, e consequente disponibilidade para a prática de atividade físicas, viram-se agravadas com o confinamento e as exigências de vida impostas pela COVID-19.

Relativamente às diferenças de gênero no que se refere ao comportamento alimentar, no contexto europeu e nos Estados Unidos, o conflito trabalho-família foi maior entre mulheres trabalhadoras em sistema de home office com filhos entre zero e cinco anos de idade (DEL BOCA et al., 2020; YILDIRIM; ESLEN-ZIYA, 2020), sobretudo se os parceiros se mantiveram no trabalho fora de casa. (DEL BOCA et al., 2020) A presença dos filhos afetou desproporcionalmente as mulheres com relação ao trabalho doméstico. Elas acumularam a carga do trabalho, os cuidados com as crianças e as tarefas domésticas, incluindo a higienização mais rigorosa e preparação dos alimentos, atividades que foram aumentadas durante o distanciamento social. (YILDIRIM; ESLEN-ZIYA, 2020) Quando houve divisão das tarefas, entre participantes da Itália, foi mais provável a participação dos homens nos cuidados com as crianças do que nas tarefas domésticas. (DEL BOCA et al., 2020) No Brasil, o Instituto Brasileiro de Geografia e Estatística (IBGE) registrou $16,4 \%$ a mais no trabalho remoto feminino do que os homens durante a pandemia no mês de julho. (IBGE, 2020)

Alguns estudos referem que em circunstâncias adversas, as mulheres tendem a aumentar o consumo de tabaco, enquanto os homens aumentam mais facilmente o consumo de álcool, estando isso relacionado com uma série de questões culturais e sociais de menor externalização por parte delas. (JAPUNTICH et al., 2016; 
TORRES; NOWSON, 2007) No estudo brasileiro de Malta e demais autores (2020), observou-se um aumento do consumo de tabaco em ambos os sexos, no entanto, quando estratificada a amostra por sexo, o percentual de aumento de 10 cigarros por dia foi maior entre as mulheres (28,9\% - IC95\%: 24,7;33,6), comparadas com os homens (16,8\% - IC95\%: 13,3;21,0). O mesmo estudo não encontrou diferenças em função do gênero no que se referiu ao aumento do consumo de bebidas alcoólicas. No âmbito da abordagem das questões de gênero e no que refere ao consumo de álcool, além dos prejuízos em relação à própria saúde, é necessário ter em atenção que o consumo excessivo se associa à violência doméstica contra a mulher, outro flagelo que aumentou com a pandemia e o confinamento em todo o mundo. (FINLAY; GILMORE, 2020)

As diferenças de gênero não se encontram apenas nos estilos de vida, mas sobretudo nos fatores psicossociais que os influenciam (PATRÃO et al., 2019a; ZANTINGE et al., 2014), que são permeados por uma série de desigualdades sociais. A vida do cotidiano e os estilos de vida não são neutros do ponto de vista do gênero e as crises, tal como esta que se está vivendo com a pandemia de COVID-19, tende a exacerbar as desigualdades existentes entre mulheres e homens. Assim, seria essencial que esta crise sanitária servisse, não para agravar esses problemas, mas para refletir sobre a necessidade de dar condições de igualdade e justiça, de forma a que homens e mulheres tenham as mesmas oportunidades e condição de vida para promoverem e cuidarem da sua saúde. 


\section{Discussão e Conclusão}

À primeira vista, esta pandemia e a resposta do mundo a ela parecem estar muito distantes das perspectivas da promoção da saúde, na medida em que os esforços e os profissionais de saúde em destaque são os que conseguem contribuir para o evitamento de um evento contagioso e aqueles que enfrentam um tsunami de pacientes que sofrem de uma potencial doença mortal, parecendo haver pouca necessidade de especialistas cuja experiência está na outra extremidade da saúde. (SPRINGER; PHILLIPS, 2006; VAN DEN BROUCKE, 2020) No entanto, a promoção da saúde e os seus princípios são mais pertinentes e essenciais do que nunca neste contexto, podendo capacitar as pessoas e os grupos para aumentarem o controle sobre a sua saúde e os determinantes que a influenciam, contribuindo, inclusive, em vários níveis: primeiramente, ajudando as pessoas a mudarem comportamentos de saúde e gerirem a doença; a um nível intermédio, promovendo intervenções de saúde a nível comunitário; e a montante, informando sobre as políticas de saúde que afetam as populações. (BROWNSON; SEILER; EYLER, 2010; VAN DEN BROUCKE, 2020)

No que se refere especificamente aos comportamentos relacionados com o estilo de vida aqui abordados, centrais na promoção da saúde, é necessário que pessoas de todas as idades e gêneros sigam as recomendações de manutenção da prática de atividade física e evitem o comportamento sedentário durante a pandemia, quer seja em ambientes domiciliares ou nos espaços permitidos por medidas oficiais em âmbito nacional e local, respeitando as orientações das autoridades sanitárias para a manutenção do distanciamento social e dos cuidados e medidas de higiene. (ALMEIDA; QUEIROZ; AQUINO, 2020; AMERICAN COLLEGE OF SPORTS MEDICINE, 2020; CHEN et al., 2020; PITANGA; BECK; PITANGA, 2020c) Recomenda-se ainda que a elaboração de políticas públicas voltadas à promoção da 
atividade física e incentivo ao estilo de vida ativo durante e pós-pandemia contemplem ações para além do discurso biológico e centrado nos sujeitos, mas que que favoreçam o empoderamento e a autonomia da sociedade para a sua prática, sendo sensíveis às especificidades de gênero e as desigualdades estruturais. (KNUTH; CARVALHO; FREITAS, 2020) Adicionalmente, é necessário centrar esforços para a melhoria do comportamento alimentar, por sua relação com as doenças crônicas não transmissíveis que interagem com a COVID-19, com consequências mais graves e sequelas permanentes, ou de longo curso, que sobrecarregarão ainda mais os serviços de saúde em um futuro próximo. No que se refere especificamente aos consumos de álcool e tabaco, as estratégias de promoção da saúde devem ser promovidas e estar próximas das pessoas, que poderão estar mais isoladas do que nunca neste momento. Adicionalmente, seria importante ter alguns cuidados ao nível da exposição mediática desses comportamentos. De acordo com Chagas; Paula; Martins (2020), referindo-se especificamente ao aumento do consumo de álcool na população brasileira, é muito importante que os meios de comunicação demandem aos especialistas esclarecimentos ou previsões sobre os impactos do isolamento social sobre o consumo de álcool, porém, tendo por base a teoria da educação normativa, é necessário ter o cuidado de não passar a mensagem de que "todo o mundo" está bebendo mais, induzindo assim as pessoas a consumirem mais álcool na tentativa de equiparação grupal dos padrões de consumo. Relativamente ao hábito de fumar, há ainda a ter em consideração que este está associado a piora no desenvolvimento da COVID-19, dado que as doenças cardiovasculares e respiratórias, além de evoluírem mais rapidamente entre fumantes, têm sido associadas a um pior prognóstico em pacientes de COVID-19. (MALTA et al., 2020; VOLKOW, 2020) Assim sendo, é necessário prestar especial atenção a esse grupo de pessoas que fumam, devido ao maior risco de evolução para formas graves da doença. (MALTA et al., 2020) 
Em suma, considera-se que faz todo o sentido manter e promover ainda mais as mensagens e estratégias de incentivo a um estilo de vida saudável, mesmo em tempos de pandemia, confinamentos e em uma conjuntura em que muitas preocupações urgentes de saúde se impõem. (CANCELLO et al., 2020) Além disso, é necessário perceber que os impactos desta pandemia são desproporcionais para as mulheres e transcendem a esfera da saúde no que se refere à COVID-19, acarretando efeitos relevantes relativamente à questão da promoção de um estilo de vida saudável.

\section{Referências}

ALMEIDA, R. T.; QUEIROZ, C. O.; AQUINO, E. M. L. Declaring Physical Activity as 'Essential' During the COVID-19 Pandemic May not be a Good Measure. International Journal of Cardiovascular Sciences, Rio de Janeiro, v. 33, n. 5, p. 589-590, 25 Aug. 2020. DOI: https://doi.org/10.36660/ijcs.20200165. Disponível em: https://www.scielo.br/scielo.php?script=sci_arttext\&pid =S2359-56472020005008206\&lng=en\&nrm=iso. Acesso em: 30 set. 2020.

ALTIERI, M. A.; NICHOLLS, C. I. Agroecology and the reconstruction of a post-COVID-19 agriculture. The Journal of Peasant Studies, London, v. 47, n. 5, p. 881-898, July 2020.

AMERICAN COLLEGE OF SPORTS MEDICINE. Staying Physically Active During the COVID-19 Pandemic. Indianapolis, 16 Mar. 2020. Disponível em: https://www.acsm. org/read-research/newsroom/news-releases/news-detail/2020/03/16/stayingphysically-active-during-covid-19-pandemic. Acesso em: 30 set. 2020.

AMMAR, A. et al. Effects of COVID-19 home confinement on eating behaviour and physical activity: Results of the ECLB-COVID19 international online survey. Nutrients, Basel, v. 12, n. 6, 2020.

BENNET, P. Introdução clínica à psicologia da saúde. Lisboa: Climepsi, 2002.

BENNET, P.; MURPHY, S. Psicologia e promoção da saúde. Lisboa: Climepsi, 1999. 
BORZEKOWSKI, D. L. G.; ROBINSON, T. N. The 30-second effect:

an experiment revealing the impact of television commercials on food preferences of preschoolers. Journal of the American Dietetic Association, Chicago, v. 101, n. 1, p. 42-46, 2001.

BRASIL. Ministerio da Saúde; Secretaria de Vigilância em Saúde. Guia alimentar para a população brasileira guia alimentar. Brasília, DF: Ministério da Saúde. Secretaria de Atenção à Saúde. Departamento de Atenção Básica, 2014. Disponível em: https://bvsms.saude.gov.br/bvs/publicacoes/guia_alimentar_ populacao_brasileira_2ed.pdf. Acesso em: 30 set. 2020.

BROWNSON, R. C.; SEILER, R.; EYLER, A. A. Measuring the impact of public health policy. Preventing Chronic Disease, Atlanta, v. 7, n. 4, p. A77, July 2010.

CANCELLO, R. et al. Determinants of the Lifestyle Changes during COVID-19 Pandemic in the Residents of Northern Italy. International Journal of Environmental Research and Public Health, Basel, v. 17, n. 17, p. 6287, Aug. 2020.

CAPONNETTO, P. et al. Smoking behavior and psychological dynamics during COVID-19 social distancing and stay-at-home policies: A survey. Health Psychology Research, Pavia, IT, v. 8, n. 1, p. 9124, May 2020.

CARVALHO, F. F. B. de; SILVA, R. G. da; OLIVEIRA, R. B. A essencialidade das academias de ginástica para a saúde diante da pandemia da COVID-19 no Brasil. Revista Brasileira de Atividade Física \& Saúde, Florianópolis, v. 25, p. 1-5, 14 set. 2020. Disponível em: https://rbafs.org.br/RBAFS/article/view/14282. Acesso em: 30 set. 2020.

CASPERSEN, C. J.; POWELL, K. E.; CHRISTENSON, G. M. Physical activity, exercise, and physical fitness: definitions and distinctions for health-related research. Public Health Reports, Rockville, v. 100, n. 2, p. 126-131, 1985.

CHAGAS, C.; PAULA, T. C. S. de; MARTINS, L. B. O aumento do consumo de álcool em tempos de pandemia: mídia e normas sociais. Comunicação em Ciências da Saúde, Brasília, DF, v. 31, n. 1, p. 116-120, 2020.

CHAU, J. Y. et al. Cross-sectional associations of total sitting and leisure screen time with cardiometabolic risk in adults. Results from the HUNT Study, Norway. Journal of Science and Medicine in Sport, Victoria, v. 17, n. 1, p. 78-84, Jan. 2014. Disponível em: https://pubmed.ncbi.nlm.nih.gov/23619159/.

Acesso em: 30 set. 2020. 
CHEN, P. et al. Coronavirus disease (COVID-19): The need to maintain regular physical activity while taking precautions. Journal of Sport and Health Science, Shanghai, v. 9, n. 2, p. 103-104, 1 Mar. 2020. Disponível em: https://pubmed.ncbi. nlm.nih.gov/32099716/. Acesso em: 30 set. 2020.

CLARKE, D. Junk Food Brand Drops Advertising to Kids During Lockdown to Avoid Formal Regulatory Investigation. Action on Salt, United Kingdom, 21 May 2020.

Disponível em: http://www.actiononsalt.org.uk/news/news/2020/news-stories/ junk-food-brand-drops-advertising-to-kids-during-lockdown-to-avoid-formalregulatory-investigation.html. Acesso em: 30 set. 2020.

CORDAIN, L. et al. Origins and evolution of the Western diet: health implications for the 21st century. The American Journal of Clinical Nutrition, Bethesda,v. 81, n. 2, p. 341-354, 1 Feb. 2005.

CORTEZ, A. C. L. et al. Centers of physical activities and health promotion during the COVID-19 pandemic. Scielo Preprints, São Paulo, 2020.

CROCHEMORE-SILVA, I. et al. Prática de atividade física em meio a pandemia da COVID-19: estudo de base populacional em cidade do sul do Brasil.

Ciência \& Saúde Coletiva, Rio de Janeiro, 2020. Disponível em: http://www. cienciaesaudecoletiva.com.br/artigos/pratica-de-atividade-fisica-em-meio-apandemia-da-covid19-estudo-de-base-populacional-em-cidade-do-sul-dobrasil/17777?id=17777. Acesso em: 30 set. 2020.

DEL BOCA, D. et al. Women's and men's work, housework and childcare, before and during COVID-19. Review of Economics of the Household, Dordrecht, 2020.

DI RENZO, L. et al. Eating habits and lifestyle changes during COVID-19 lockdown: an Italian survey. Journal of Translational Medicine, London, v. 18, n. 1, p. 229, 8 Dec. 2020a.

DI RENZO, L. et al. Psychological Aspects and Eating Habits during COVID-19 Home Confinement: Results of EHLC-COVID-19 Italian Online Survey. Nutrients, Basel, v. 12, n. 7, July 2020b.

DIETZ, W.; SANTOS-BURGOA, C. Obesity and its Implications for COVID-19 Mortality. Obesity, Silver Spring, v. 28, n. 6, p. 1005-1005, 18 June. 2020.

ĐOGAŠ, Z. et al. The effect of COVID-19 lockdown on lifestyle and mood in Croatian general population: a cross-sectional study. Croatian Medical Journal, Zagreb, v. 61, n. 4, p. 309-318, 31 Aug. 2020. 
DURAN, P.; CABALLERO, B.; ONIS, M. The Association between Stunting and Overweight in Latin American and Caribbean Preschool Children. Food and Nutrition Bulletin, Tokyo, v. 27, n. 4, p. 300-305, 10 Dec. 2006.

FINLAY, I.; GILMORE, I. Covid-19 and alcohol-a dangerous cocktail. BMJ (Clinical research ed.), London, v. 369, p. m1987, 20 May2020.

FITBIT. The impact of coronavirus on global activity. 23 Mar. 2020. Disponível em: https://blog.fitbit.com/covid-19-global-activity/. Acesso em: 19 set. 2020.

GALASSO, V. et al. Gender Differences in COVID-19 Related Attitudes and Behavior: Evidence from a Panel Survey in Eight OECD Countries. Cambridge, 2020. Disponível em: https://www.nber.org/system/files/working_papers/w27359/ w27359.pdf. Acesso em: 1 out. 2020.

GARCÍA-ÁLVAREZ, L. et al. ¿Se observarán cambios en el consumo de alcohol y tabaco durante el confinamiento por COVID-19? Adicciones, Barcelona, v. 32, n. 2, p. 85-89, 1 Apr. 2020.

GOMES, C. M. et al. Impact of COVID-19 on clinical practice, income, health and lifestyle behavior of Brazilian urologists. International Braz J Urol, Rio de Janeiro, v. 46, n. 6, p. 1042-1071, dez. 2020.

GREEN, W. D.; BECK, M. A. Obesity Impairs the Adaptive Immune Response to Influenza Virus. Annals of the American Thoracic Society, New York, v. 14, supl. 5, p. S406-S409, Nov. 2017.

HAMER, M.; STAMATAKIS, E.; STEPTOE, A. Effects of substituting sedentary time with physical activity on metabolic risk. Medicine and Science in Sports and Exercise, Madison, v. 46, n. 10, p. 1946-1950, 2014. Disponível em: https://pubmed.ncbi.nlm. nih.gov/24674977/. Acesso em: 30 set. 2020.

HANKIVSKY, O. Women's health, men's health, and gender and health: Implications of intersectionality. Social Science \& Medicine, Oxford, v. 74, n. 11, p. 1712-1720, June. 2012.

HARTUNG, P. A. D.; KARAGEORGIADIS, E. V. A regulação da publicidade de alimentos e bebidas não alcoólicas para crianças no Brasil. Revista de Direito Sanitário, São Paulo, v. 17, n. 3, p. 160, 2017.

IBGE. Pesquisa Nacional por amostra de municípios - PNAD COVID19: resultado mensal. Rio de Janeiro: IBGE, 2020. Disponível em: https://biblioteca.ibge.gov.br/ visualizacao/livros/liv101745.pdf. Acesso em: 1 out. 2020. 
JACKSON, S. E. et al. Association of the Covid-19 lockdown with smoking, drinking, and attempts to quit in England: an analysis of 2019-2020 data. MedRxiv, [s. I.], p. 1-24, 2020.

JAPUNTICH, S. J. et al. Deployment stress, tobacco use, and postdeployment posttraumatic stress disorder: Gender differences. Psychological Trauma: theory, research, practice, and policy, Washington, v. 8, n. 2, p. 123-126, 2016.

JIMÉNEZ-PAVÓN, D.; CARBONELL-BAEZA, A.; LAVIE, C. J. Physical exercise as therapy to fight against the mental and physical consequences of COVID-19 quarantine: Special focus in older people. Progress in Cardiovascular Diseases, Philadelphia, v. 63, n. 3, p. 386-388, May /June 2020. Disponível em: https:// pubmed.ncbi.nlm.nih.gov/32220590/. Acesso em: 30 set. 2020.

JONSDOTTIR, I. H. et al. A prospective study of leisure-time physical activity and mental health in Swedish health care workers and social insurance officers. Preventive Medicine, San Diego, v. 51, n. 5, p. 373-377, Nov. 2010. Disponível em: https://pubmed.ncbi.nlm.nih.gov/20691721/. Acesso em: 30 set. 2020.

KHAN, M. A.; MOVERLEY SMITH, J. E. "Covibesity," a new pandemic. Obesity Medicine, [Amsterdam], v. 19, n. 2, p. 100282, Sept. 2020.

$\mathrm{KIS}$, O. et al. Minimally supervised home-based resistance training and muscle function in older adults: A meta-analysis. Archives of Gerontology and Geriatrics, Amsterdam, v. 84, Sept./Oct. 2019. Disponível em: https://pubmed.ncbi.nlm.nih. gov/31301519/. Acesso em: 30 set. 2020.

KNUTH, A. G.; CARVALHO, F. F. B. de; FREITAS, D. D. Discursos de instituições de saúde brasileiras sobre atividade física no início da pandemia de COVID-19. Revista Brasileira de Atividade Física \& Saúde, Florianópolis, v. 25, p. 1-9, 14 set. 2020. Disponível em: https://rbafs.org.br/RBAFS/article/view/14342. Acesso em: 30 set. 2020.

KOHUT, M. L. et al. Exercise and psychosocial factors modulate immunity to influenza vaccine in elderly individuals. Journals of Gerontology. Series A Biological Sciences and Medical Sciences, Washington, v. 57, n. 9, p. 557-562, 2002.

Disponível em: https://pubmed.ncbi.nlm.nih.gov/12196490/. Acesso em: 30 set. 2020.

KRINSKI, K. et al. Efeitos do exercício físico no sistema imunológico. Revista Brasileira de Medicina, São Paulo, v. 67, n. 7, p. 228-233, 2010. 
LACAILLE, L. et al. Eating Behavior. In: GELLMAN, M.D., TURNER, J. R. (ed.). Encyclopedia of Behavioral Medicine. New York: Springer New York, 2013. p. 641-642. Disponível em: http://link.springer.com/10.1007/978-1-4419-10059_1613. Acesso em: 17 set. 2020.

LADDU, D. R. et al. Physical activity for immunity protection: Inoculating populations with healthy living medicine in preparation for the next pandemic. Progress in Cardiovascular Diseases, Philadelphia, p. 1-3, Apr. 2020. Disponível em: https://pubmed.ncbi.nlm.nih.gov/32278694/. Acesso em: 30 set. 2020.

LEBEAUT, A.; TRAN, J. K.; VUJANOVIC, A. A. Posttraumatic stress, alcohol use severity, and alcohol use motives among firefighters: The role of anxiety sensitivity. Addictive Behaviors, Oxford, v. 106, p. 106353, July 2020.

LEE, B. et al. Effects of exposure to television advertising for energy-dense/ nutrient-poor food on children's food intake and obesity in South Korea. Appetite, London, v. 81, p. 305-311, 2014. Disponível em: http://dx.doi.org/10.1016/j. appet.2014.06.103. Acesso em: 29 set. 2020.

LIN, X. et al. Leisure time physical activity and cardio-metabolic health: Results from the Brazilian longitudinal study of adult health (ELSA-Brasil). Journal of the American Heart Association, Oxford, v. 5, n. 6, p. e003337, 1 jun. 2016. Disponível em: https://pubmed.ncbi.nlm.nih.gov/27412901/. Acesso em: 30 set. 2020.

LÓPEZ DE ROMAÑA, D.; OLIVARES, M.; BRITO, A. Introduction: Prevalence of Micronutrient Deficiencies in Latin America and the Caribbean. Food and Nutrition Bulletin, Tokyo, v. 36, supl. 2, p. S95-S97, June 2015.

LUZI, L.; RADAELLI, M. G. Influenza and obesity: its odd relationship and the lessons for COVID-19 pandemic. Acta Diabetologica, Berlin, v. 57, n. 6, p. 759-764, 1 June 2020. Disponivel em: https://pubmed.ncbi.nlm.nih.gov/32249357/. Acesso em: 30 set. 2020.

MACHT, M. How emotions affect eating: A five-way model. Appetite, London, v. 50, n. 1, p. 1-11, Jan. 2008.

MALTA, D. C. et al. A pandemia da COVID-19 e as mudanças no estilo de vida dos brasileiros adultos: um estudo transversal, 2020. Epidemiologia e Serviços de Saúde, Brasília, DF, v. 29, n. 4, 2020. Disponível em: http://www.scielo.br/ scielo.php?script=sci_arttext\&pid=S2237-96222020000400315\&tlng=pt. Acesso em: 30 set. 2020. 
MARIJN STOK, F. et al. Dietary Behavior: an interdisciplinary conceptual analysis and taxonomy. Frontiers In Psychology, Switzerland, v. 9, p. 1689, 2018. Disponível em: http://www.ncbi.nlm.nih.gov/pubmed/30298030. Acesso em: 28 set. 2020

MONTEIRO, C. A. et al. Ultra-processed foods, diet quality, and health using the NOVA classification system. Roma: FAO, 2019. Disponível em: www.fao.org/3/ ca5644en/ca5644en.pdf. Acesso em: 28 set. 2020.

MORAES, A. C. F. de et al. Independent and Combined Effects of Physical Activity and Sedentary Behavior on Blood Pressure in Adolescents: Gender Differences in Two Cross-Sectional Studies. PLoS ONE, California, v. 8, n. 5, p. e62006, 1 May 2013. Disponível em: https://pubmed.ncbi.nlm.nih.gov/23650506/. Acesso em: 30 set. 2020.

NAJA, F.; HAMADEH, R. Nutrition amid the COVID-19 pandemic: a multi-level framework for action. European Journal of Clinical Nutrition, London, v. 74, n. 8 , p. 1117-1121, 20 Aug. 2020.

NIELSEN, G. COVID-19: tracking the impact 2020. The Nielsen Company, New York, May 2020. Disponível em: https://www.nielsen.com/us/en/insights/article/2020/ covid-19-tracking-the-impact-on-fmcg-and-retail/. Acesso em: 1 out. 2020.

NIEMAN, D. C. Coronavirus disease-2019: A tocsin to our aging, unfit, corpulent, and immunodeficient society. Journal of Sport and Health Science, Shanghai, v. 9, n. 4, p. 293-301, 1 jul. 2020. Disponível em: https://pubmed.ncbi.nlm.nih. gov/32389882/. Acesso em: 30 set. 2020.

OGDEN, J. Psicologia da saúde. 2. ed. Lisboa: Climepsi, 2004.

OLIVEIRA NETO, L. et al. Coronavirus Pandemic (SARS-COV-2): Pre-Exercise Screening Questionnaire (PESQ) for Telepresential Exercise. Frontiers in Public Health, Lausanne, v. 8, n. 146, 21 Apr. 2020. Disponível em: https://www.ncbi.nlm. nih.gov/pmc/articles/PMC7186444/. Acesso em: 30 set. 2020.

PATE, R. R.; O'NEILL, J. R.; LOBELO, F. The evolving definition of "sedentary". Exercise and Sport Sciences Reviews, v. 36, n. 4, p. 173-178, Oct. 2008. Disponível em: https://pubmed.ncbi.nlm.nih.gov/18815485/. Acesso em: 30 set. 2020.

PATRÃO, A. L. et al. Association Between Perceived Discrimination and Alcohol and Tobacco Consumption in ELSA-Brasil Cohort: Focusing on Gender Differences. Substance Use \& Misuse, London, v. 54, n. 7, p. 1214-1225, 7 June 2019a. 
PATRÃO, A. L. et al. Gender and psychosocial factors associated with healthy lifestyle in the Brazilian Longitudinal Study of Adult Health (ELSA-Brasil) cohort: a cross-sectional study. BMJ Open, London, v. 7, n. 8, p. e015705, 28 Aug. 2017.

PATRÃO, A. L. et al. Health behavior-related indicator of lifestyle: application in the ELSA-Brasil study. Global Health Promotion, London, v. 26, n. 4, p. 62-69, 11 dez. 2019b.

PEARCE, N. et al. The road to $25 \times 25$ : How can the five-target strategy reach its goal? The Lancet Global Health, England, v. 2, n. 3, p. 126-128, 2014.

PEIXOTO, H.; LANDAU, L. Com delivery, pequenos agricultores orgânicos driblam crise e veem até aumento de vendas na pandemia. BBC News Brasil, Rio de Janeiro, 11 jun. 2020. Disponível em: https://www.bbc.com/portuguese/geral-53357892. Acesso em: 30 set. 2020.

PIGGIN, J. What Is Physical Activity? A Holistic Definition for Teachers, Researchers and Policy Makers. Frontiers in Sports and Active Living, Switzerland, v. 2, 18 June 2020.

PINHO, C. S. et al. The effects of the COVID-19 pandemic on levels of physical fitness. Revista da Associação Médica Brasileira, São Paulo, v. 66, supl. 2, p. 34-37, 2020. Disponível em: http://www.scielo.br/scielo.php?script=sci_arttext\& pid=S0104-42302020001400034\&tIng=en. Acesso em: 30 set. 2020.

PITANGA, C. P. S.; BECK, C. C.; PITANGA, F. J. G. Home-Based Physical Exercise Program for Cardiac Rehabilitation in the Context of COVID-19. Journal of Heart Health, Milpitas, CA, v. 6, n. 1, 2020. Disponível em: https://www.sciforschenonline. org/journals/heart/JHH156.php. Acesso em: 1 out. 2020.

PITANGA, F. J. G. et al. Association between leisure-time physical activity and sedentary behavior with cardiometabolic health in the ELSA-Brasil participants. SAGE Open Medicine, London, v. 7, p. 1-9, jan. 2019. Disponível em: /pmc/articles/ PMC6354306/?report=abstract. Acesso em: 30 set. 2020.

PITANGA, F. J. G.; BECK, C. C.; PITANGA, C. P. S. Atividade física e redução do comportamento sedentário durante a pandemia do coronavírus. Arquivos Brasileiros de Cardiologia, Rio de Janeiro, v. 114, n. 6, p. 1058-1060, 8 maio 2020a. Disponível em: https://www.scielo.br/scielo.php?script=sci_arttext\&pid=S0066-782 X2020005007201\&lng=pt\&nrm=iso\&tlng=pt. Acesso em: 30 set. 2020.

PITANGA, F. J. G.; BECK, C. C.; PITANGA, C. P. S. Inatividade física, obesidade e COVID-19: perspectivas entre múltiplas pandemias. Revista Brasileira de Atividade Física \& Saúde, Florianópolis, v. 25, p. 1-4, 2020b. 
PITANGA, F. J. G.; BECK, C. C.; PITANGA, C. P. S. Should Physical Activity Be Considered Essential During the COVID-19 Pandemic? International Journal of Cardiovascular Sciences, Rio de Janeiro, v. 33, n. 4, p. 401-403, 1 June 2020c. Disponível em: https://www.scielo.br/scielo.php?script=sci_arttext\&pid $=$ =S2359-56472020005005201\&Ing=en\&nrm=iso\&tlng=en. Acesso em: 30 set. 2020.

RAMOS, F. A crise da pandemia, os modelos de produção animal e a produção orgânica como alternativa viável. Portal do Instituto Brasil Orgânico, Rio de Janeiro, 10 abr. 2020. Disponível em: https://institutobrasilorganico.org/2020/07/a-criseda-pandemia-os-modelos-de-producao-animal-e-a-producao-organica-comoalternativa/. Acesso em: 30 set. 2020.

READ, J. G.; GORMAN, B. K. Gender and Health Inequality. Annual Review of Sociology, v. 36, n. 1, p. 371-386, June 2010.

RODRIGUES, P. et al. Fatores associados a pratica de atividades físicas durante a pandemia da COVID-19 no estado do Rio de Janeiro, Brasil. Revista Brasileira de Atividade Física \& Saúde, Florianópolis, v. 25, p. 1-9, 23 Sept. 2020. Disponível em: https://rbafs.org.br/RBAFS/article/view/14365. Acesso em: 30 set. 2020.

RUIZ-ROSO, M. B. et al. Covid-19 confinement and changes of adolescent's dietary trends in Italy, Spain, Chile, Colombia and Brazil. Nutrients, Basel, v. 12, n. 6, p. 1-18, 2020.

RUNDLE, A. G. et al. COVID-19-Related School Closings and Risk of Weight Gain Among Children. Obesity, Malden, 28, n. 6, p. 1008-1009, 18 June 2020.

SCHULER, P. B.; LEBLANC, P. A.; MARZILLI, T. S. Effect of physical activity on the production of specific antibody in response to the 1998-99 influenza virus vaccine in older adults. Journal of Sports Medicine and Physical Fitness, Tokyo, v. 43, n. 3, p. 404, 2003.

SIDOR, A.; RZYMSKI, P. Dietary Choices and Habits during COVID-19 Lockdown: Experience from Poland. Nutrients, Basel, v. 12, n. 6, p. 1657, 3 June 2020.

SIU, E.; CAMPITELLI, M. A.; KWONG, J. C. Physical activity and influenza-coded outpatient visits, a population-based cohort study. PLoS ONE, California, v. 7, n. 6, p. e39518, 21 jun. 2012. Disponível em: https://pubmed.ncbi.nlm.nih. gov/22737242/. Acesso em: 30 set. 2020. 
SOLON JÚNIOR, L. J. F. et al. Home-based exercise during confinement in COVID-19 pandemic and mental health in adults: a cross-sectional comparative study. Revista Brasileira de Atividade Física \& Saúde, Florianópolis, v. 25, p. 1-7, 14 set. 2020. Disponível em: https://rbafs.org.br/RBAFS/article/view/14336. Acesso em: 30 set. 2020.

SPENCER, S. H. False claims of nationwide lockdown for COVID-19. FactCheck.org, Philadelphia, Mar. 2020. Disponível em: https://www.factcheck.org/2020/03/falseclaims-of-nationwide-lockdown-for-covid-19/. Acesso em: 17 set. 2020.

SPRINGER, F; PHILLIPS, J. L. The IOM model: a tool for prevention planning and implementation. Prevention Tactics,[s. I.], v. 8, n. 13, p. 1-7, 2006. Disponível em: https://www.naspa.org/images/uploads/kcs/WHPL_Canon_Pr_ Prevention_Tactics_Springer_8.pdf. Acesso em: 30 set. 2020.

SUN, Y. et al. Brief Report: increased addictive internet and substance use behavior during the COVID-19 pandemic in China. The American Journal on Addictions, Oxford, v. 29, n. 4, p. 268-270, 4 July 2020.

TAKEDA, E. et al. Stress control and human nutrition. The Journal of Medical Investigation, Tokushima, v. 51, n. 3/4, p. 139-145, 2004.

THE LANCET GASTROENTEROLOGY \& HEPATOLOGY. Drinking alone: COVID-19, lockdown, and alcohol-related harm. The Lancet Gastroenterology \& Hepatology, Amsterdam, v. 5, n. 7, p. 625, July 2020.

THE NIELSEN COMPANY. Advertising in the Age of COVID-19. New York, 2020.

TORRES, S. J.; NOWSON, C. A. Relationship between stress, eating behavior, and obesity. Nutrition, Tarrytown, v. 23, n. 11/12, p. 887-894, Nov. 2007.

TREMBLAY, M. S. et al. Sedentary Behavior Research Network (SBRN) Terminology Consensus Project process and outcome. International Journal of Behavioral Nutrition and Physical Activity, London, v. 14, n. 1, p. 1-17, 10 June 2017. Disponível em: https://pubmed.ncbi.nlm.nih.gov/28599680/. Acesso em: 30 set. 2020.

VAN DEN BROUCKE, S. Why health promotion matters to the COVID-19 pandemic, and vice versa. Health Promotion International, Oxford,v. 35, n. 2, p. 181-186, 1 Apr. 2020. 
VAN DER PLOEG, H. P. et al. Sitting time and all-cause mortality risk in 222 497 Australian adults. Archives of Internal Medicine, Chicago, v. 172, n. 6, p. 494-500, 26 Mar. 2012. Disponível em: https://jamanetwork.com/journals/ jamainternalmedicine/fullarticle/1108810. Acesso em: 30 set. 2020.

VERMA, S.; MISHRA, A. Depression, anxiety, and stress and socio-demographic correlates among general Indian public during COVID-19. International Journal of Social Psychiatry, London, v. 66, n. 8, p. 756-762, 20 Dec. 2020.

VOLKOW, N. D. Collision of the COVID-19 and Addiction Epidemics. Annals of Internal Medicine, Philadelphia, v. 173, n. 1, p. 61-62, 7 July 2020.

WONG, C. M. et al. Is exercise protective against influenza-associated mortality? PLoS ONE, California, v. 3, n. 5, p. e2108, 7 May 2008. Disponível em: https:// pubmed.ncbi.nlm.nih.gov/18461130/. Acesso em: 30 set. 2020.

WOODS, J. A. et al. Cardiovascular exercise training extends influenza vaccine seroprotection in sedentary older adults: The immune function intervention trial. Journal of the American Geriatrics Society, Malden, v. 57, n. 12, p. 2183-2191, Dec. 2009. Disponível em: https://pubmed.ncbi.nIm.nih. gov/20121985/. Acesso em: 30 set. 2020.

WORLD HEALTH ORGANIZATION -WHO. Food and nutrition tips during selfquarantine. [S. I.], 2020a. Disponível em: https://www.euro.who.int/en/healthtopics/health-emergencies/coronavirus-covid-19/technical-guidance/food-andnutrition-tips-during-self-quarantine. Acesso em: 30 set. 2020.

WORLD HEALTH ORGANIZATION - WHO. Infection prevention and control guidance - (COVID-19). [S. I.], 2020b. Disponível em: https://www.who.int/ westernpacific/emergencies/covid-19/technical-guidance/infection-preventioncontrol. Acesso em: 30 set. 2020.

WORLD HEALTH ORGANIZATION - WHO. Stay physically active during selfquarantine. [S. I.], 2020c. Disponível em: https://www.euro.who.int/en/healthtopics/health-emergencies/coronavirus-covid-19/technical-guidance/stayphysically-active-during-self-quarantine. Acesso em: 30 set. 2020c.

WRIGHT, L.; STEPTOE, A.; FANCOURT, D. Are we all in this together? Longitudinal assessment of cumulative adversities by socioeconomic position in the first 3 weeks of lockdown in the UK. Journal of Epidemiology and Community Health, London, v. 74, n. 9, p. 683-688, 2020. 
WU, P. et al. Alcohol Abuse/Dependence Symptoms Among Hospital Employees Exposed to a SARS Outbreak. Alcohol and Alcoholism, Oxford, v. 43, n. 6, p. 706-712, Nov./Dec. 2008.

YACH, D. Tobacco Use Patterns in Five Countries During the COVID-19 Lockdown. Nicotine \& Tobacco Research, Abingdon, v. 22, n. 9, p. 1671-1672, 24 Aug. 2020.

YILDIRIM, T. M.; ESLEN-ZIYA, H. The differential impact of COVID-19 on the work conditions of women and men academics during the lockdown. Gender, Work and Organization, Oxford, p. 1-7, 2020.

ZANTINGE, E. M. et al. Retirement and a healthy lifestyle: opportunity or pitfall? A narrative review of the literature. European Journal of Public Health, Oxford, v. 24, n. 3, p. 433-439, June 2014. 